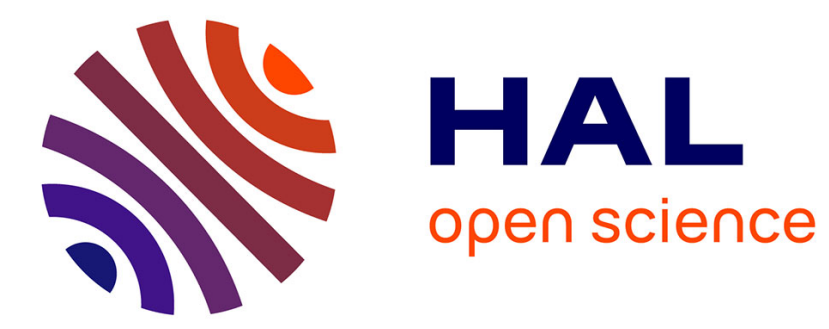

\title{
Rapid Plateau border size variations expected in three simple experiments on $2 \mathrm{D}$ liquid foams
}

\author{
Cyprien Gay, Pierre Rognon, Douglas Reinelt, François Molino
}

\section{To cite this version:}

Cyprien Gay, Pierre Rognon, Douglas Reinelt, François Molino. Rapid Plateau border size variations expected in three simple experiments on 2D liquid foams. European Physical Journal E: Soft matter and biological physics, 2011, 34 (1), pp.1-11. 10.1140/epje/i2011-11002-3 . hal-00361004

\section{HAL Id: hal-00361004 https://hal.science/hal-00361004}

Submitted on 12 Feb 2009

HAL is a multi-disciplinary open access archive for the deposit and dissemination of scientific research documents, whether they are published or not. The documents may come from teaching and research institutions in France or abroad, or from public or private research centers.
L'archive ouverte pluridisciplinaire HAL, est destinée au dépôt et à la diffusion de documents scientifiques de niveau recherche, publiés ou non, émanant des établissements d'enseignement et de recherche français ou étrangers, des laboratoires publics ou privés. 


\title{
Plateau border bulimia transition: discontinuities expected in three simple experiments on $2 \mathrm{D}$ liquid foams
}

\author{
Pierre Rognon, François Molino, and Cyprien Gaył \\ Centre de Recherche Paul Pascal, CNRS, UPR 8641, \\ Université de Bordeaux 1, 115 Av. Schweitzer, F-33600 PESSAC, France \\ Department of Endocrinology, Institute of Functional Genomics, CNRS, \\ UMR 5203, INSERM U661, Universities of Montpellier 1 and 2, \\ 141 rue de la Cardonille, F-34094 MONTPELLIER cedex 05, France \\ Matière et Systèmes Complexes, Université Paris Diderot-Paris 7, CNRS, UMR 7057, \\ Bâtiment Condorcet, Case courrier 7056, F-75205 PARIS cedex 13, France
}

(Dated: February 12, 2009)

\begin{abstract}
We describe the geometry of foams squeezed between two solid plates (2D GG foams) in two main asymptotic regimes: fully dry floor tiles and dry pancakes. We predict an abrupt transition between both regimes, with a substantial change in the Plateau border radius. This should be observable in different types of experiments on such 2D GG foams: when foam is being progressively dried or wetted, when it is being squeezed further or stretched, when it coarsens through film breakage or Oswald ripening.
\end{abstract}

PACS numbers: 47.20.Dr, 83.80.Iz, 47.57.Bc, 68.03.Cd

\section{INTRODUCTION}

As a physical system, liquid foams display many interesting properties 11,2, 3]. Among these, 2D foams attract a widespread interest : their local structure is more easily observable than that of three-dimensional foams, and more amenable to theoretical calculations.

In the present work, we present a comprehensive (though approximate) geometric description of $2 \mathrm{D}$ foams squeezed between two solid plates ("GG" foams). A thorough examination of these geometric properties suggests non-trivial consequences which should be observable through simple experimental protocols.

This approach is extended to variational properties like stresses and dilatancy in a separate work 4 , 5].

In Section II, we will go through an original and detailed geometrical description of a $2 \mathrm{D}$ GG foam. In Section III. we will highlight, as a surprising consequence, the existence of abrupt variations of the Plateau border radius with tunable parameters of the foam, and describe three different experimental situations where such variations should be observable.

\section{2D GLASS-GLASS FOAM GEOMETRICAL VADEMECUM}

In the present section, we shall provide a geometrical description and some corresponding results for twodimensional foams squeezed between two glass plates. More specifically, the calculations are conducted for ordered, monodisperse foams. Of course, if some local disorder is present, the results should not be affected tremen-

*Electronic address: cyprien.gay@univ-paris-diderot.fr dously. But the foam should nevertheless be spatially homogeneous: there should be no large-scale gradient in cell thickness, bubble volume, etc. Indeed, such a gradient would generate subtle effects related to the osmotic pressure and force balances, which would feed the discussion substantially.

In the remaining of this article, we call $P$ the typical perimeter of the bubble, defined as the perimeter of the rounded polygon that constitutes the bubble, as seen from above, and $R$ the corresponding radius of curvature of the Plateau border (see Fig. 1, left). We call $H$ the distance between both solid plates, and $R_{\mathrm{ps}}$ the radius of curvature of the pseudo Plateau borders (see Fig. 11, bottom right). We also call $\Omega$ the bubble volume, and $\Omega_{\text {liq }}$ the volume of liquid per bubble. In the monodisperse case, the liquid volume fraction $\phi$ thus verifies:

$$
\phi=\frac{\Omega_{\mathrm{liq}}}{\Omega+\Omega_{\mathrm{liq}}}, \quad \text { i.e. } \quad \Omega_{\mathrm{liq}}=\Omega \frac{\phi}{1-\phi} .
$$

We assume that $\Omega$ remains constant: we target experimental conditions under which the applied stresses are not sufficient to compress the gas phase significantly. This is the case at atmospheric pressure, unless the bubble size is on the order of a micron or smaller.

\section{A. Geometry: floor tile versus pancake regime}

The main contributions to the quantity of liquid per bubble are pictured on Fig. 1 1 and can be calculated from simple geometrical arguments:

$$
\Omega_{\mathrm{liq}} \simeq(2-\pi / 2) P R_{\mathrm{ps}}^{2}+(2 \sqrt{3}-\pi) R^{2} H
$$

The first term corresponds to the volume of the pseudo Plateau borders. As seen from above, they correspond to the light grey regions in Fig. 1. Each portion of their 


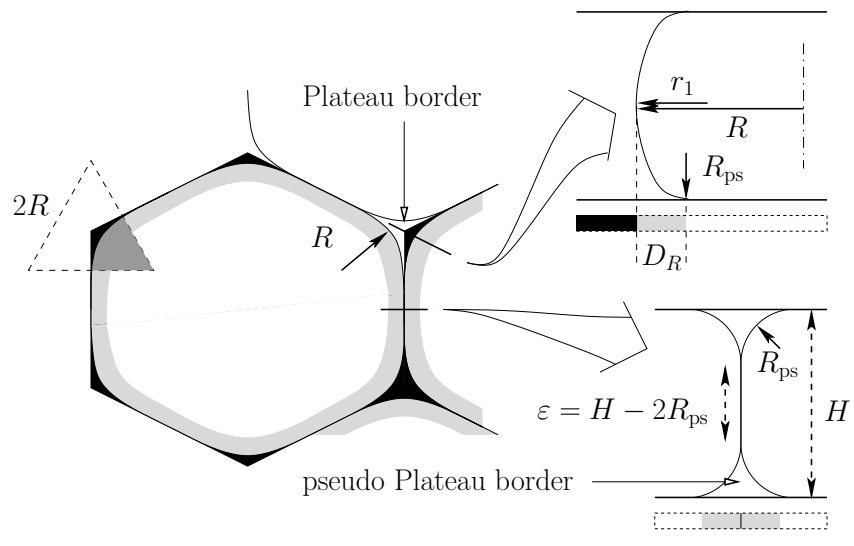

FIG. 1: Pancake conformation of a bubble squeezed between two solid plates (distance $H$ ): approximate geometry. Left: top view. The variable $P$ denotes the average perimeter of the bubbles in such a top view (outer perimeter of the light grey region). The variable $\Omega$ is the volume of the bubble gas (full thickness of the white region and part of the thickness of the light grey region), and $\mathcal{A}$ is defined as $\mathcal{A}=\Omega / H$. The variable $\mathcal{A}_{\text {tot }}$ is the total (gas and liquid) projected surface area per bubble (white and light grey and black regions). Right: two different (magnified) cross-sections (side views) with matching greyscale. As seen from above, the contact between two bubbles, for an ordered monodisperse foam, is typically along a straight line (left). In this region, the pseudo Plateau border (see bottom right drawing) has a uniform curvature of radius $R_{\mathrm{ps}}$. By contrast, in the genuine Plateau border region (left drawing), the section of the gas-liquid interface is approximately elliptical in shape (top right drawing), with principal radii of curvature $r_{1}$ and $R$ at mid-height, and $R_{\mathrm{ps}}$ and zero at the plates. The width $D_{R}$ of the curved region is intermediate between $R_{\mathrm{ps}}$ and $H / 2$ while $r_{1}$ is larger than $H / 2$.

interfaces has the shape of a quarter of a circular cylinder (see Fig. 1, bottom right). The second term in Eq. (2) corresponds to the genuine Plateau borders (black regions in Fig. 11).

Eq. (2) indicates that the squeezed 2D foam lies between two asymptotic regimes depending on volume fraction and geometry. They are pictured on Fig. 2.

1. When the Plateau border radius $R$ is much larger than the sample thickness $H$ (regimes AB and CD of Fig. 2), each bubble takes the form of a thick "pancake", and its edge is like a half cylinder with radius $H / 2$, which is the largest accessible value for the radius of the pseudo Plateau borders (see paragraph IIB below for a finer description).

2. In the reverse limit, the bubbles are shaped more like "floor tile", with sharp edges (regime E of Fig. 2): this time, the Plateau borders are like fine threads pinned on both solid plates, and each pseudo Plateau borders resembles a stretched, fine thread, glued on one of the solid plate and joining the attachment points of two Plateau borders; in

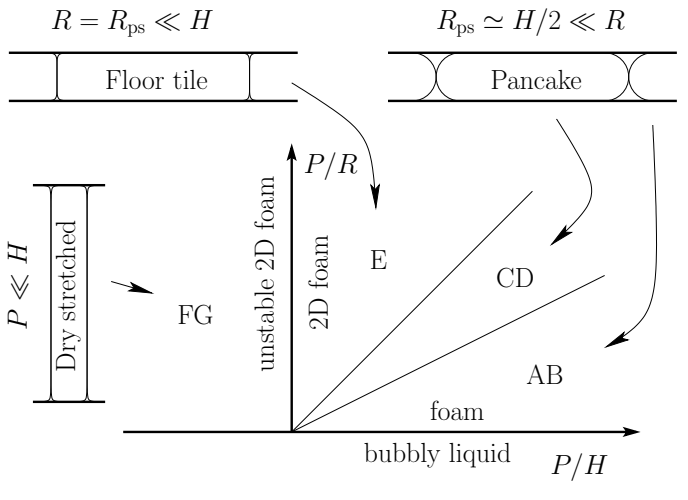

FIG. 2: Log-log representation of the regimes of a glass-glass $2 \mathrm{D}$ foam with low liquid fraction $(\phi \ll 1)$, in terms of the bubble perimeter $P$, the Plateau border radius $R$ and the cell height $H$.

The bubble perimeter $P$ is measured at mid-height of the cell: it is the outer perimeter of the light grey ribbon in Fig. 1. Such a foam can be found in two main configurations. In the floor tile situation (regimes $\mathrm{E}$ and FG) the pseudo Plateau borders are much thinner than the cell height $\left(R=R_{\mathrm{ps}} \ll H\right)$. By contrast, in the pancake regime (AB and $\mathrm{CD}$ ), although the overall liquid volume fraction $\phi$ is still much smaller than 1, facing pseudo Plateau borders almost join $\left(H-2 R_{\text {ps }} \ll H \ll R\right)$, and each bubble has a pancakelike shape. More precisely, it is useful to subdivide the pancake situation into regimes $\mathrm{AB}$ and $\mathrm{CD}$ defined by Table If: in regime $\mathrm{AB}$ (resp. $\mathrm{CD}$ ), the liquid content of the Plateau border (resp. pseudo Plateau border) dominates. The corresponding expressions for the liquid volume fraction and for several geometrical quantities are indicated in Table II. Note that regime $\mathrm{FG}$, which starts with $P \approx H$, corresponds to a non-realistic $2 \mathrm{D}$ GG foam, where the height is larger than the perimeter. The limit of a truly two-dimensional foam is obtained with $H \rightarrow \infty$. Note also that when $P=2 \pi R$ (horizontal axis), the bubbles become independent in the liquid.

this case, pseudo and genuine Plateau border radii coincide.

On Fig. 2, we have also pictured regime FG, which does not correspond to realistic $2 \mathrm{D}$ GG foams: it is useful to obtain the limit of ideal 2D foams, for which the solid plates are so far apart that the volume of the pseudo Plateau borders can be entirely neglected. This regime, although unrealistic, bridges the gap between 2D GG foam considered here and ideal $2 \mathrm{D}$ foams. This is useful in particular for discussing static dilatancy [4, 5, 6, 7].

In the pancake regimes ( $\mathrm{AB}$ and $\mathrm{CD})$, one has $R_{\mathrm{ps}} \simeq$ $H / 2$ and hence:

$$
\Omega_{\mathrm{liq}}^{\mathrm{ABCD}} \simeq(1 / 2-\pi / 8) P H^{2}+(2 \sqrt{3}-\pi) R^{2} H
$$

In regime $\mathrm{AB}$, the Plateau border contribution dominates (black regions on Fig. 11). Hence:

$$
\Omega_{\mathrm{liq}}^{\mathrm{AB}} \simeq(2 \sqrt{3}-\pi) R^{2} H
$$




\begin{tabular}{|c|c|c|c|}
\hline Regime & \multicolumn{3}{|l|}{ Conditions } \\
\hline $\mathrm{AB}$ & $\begin{array}{c}R<P \\
\left(\phi<\phi_{c}\right)\end{array}$ & $\begin{array}{c}R^{2}>P H \\
\text { (dominant Plateau borders }\end{array}$ & \\
\hline $\mathrm{CD}$ & (dominant & $\begin{array}{l}\quad R^{2}<P H \\
\text { t pseudo-Plateau borders) }\end{array}$ & $\begin{array}{c}R>H \\
\text { (pancake) }\end{array}$ \\
\hline $\mathrm{E}$ & $\begin{array}{c}R<H \\
\text { (floor tile) }\end{array}$ & $\begin{array}{c}P>H \\
(2 \mathrm{D})\end{array}$ & \\
\hline FG & $\begin{array}{r}\quad R<H \\
\text { (stretched }\end{array}$ & \begin{tabular}{|c|c}
$H$ & $P>R$ \\
$2 \mathrm{D})$ & $\left(\phi<\phi_{c}\right)$
\end{tabular} & \\
\hline
\end{tabular}

TABLE I: Four regimes for a 2D glass-glass foam. Regimes $\mathrm{AB}$ and $\mathrm{CD}$ correspond to pancake-shaped bubbles, while regime $\mathrm{E}$ corresponds to a foam made of floor tile shaped bubbles. In regimes $\mathrm{CD}$ and $\mathrm{E}$, most of the liquid is located in the pseudo Plateau borders, while in regime AB, the Plateau borders themselves have a greater volume. Regime FG does not correspond to stable 2D GG foams (they would destabilize into 3D foams), but the limit $H \rightarrow \infty$ corresponds to an ideal, truly two-dimensional foam.

Conversely, in regime CD, the pseudo Plateau borders (light grey regions on the top-view in Fig. 1) contain most of the liquid:

$$
\Omega_{\text {liq }}^{\mathrm{CD}} \simeq(1 / 2-\pi / 8) P H^{2}
$$

In regimes $\mathrm{E}$ and $\mathrm{FG}$ ( $R \ll H$, floor tile regime), the radius of curvature $R_{\mathrm{ps}}$ of the pseudo Plateau borders is equal to that of the Plateau borders:

$$
R_{\mathrm{ps}}^{\mathrm{EFG}}=R
$$

and Eq. (2) reduces to:

$$
\Omega_{\mathrm{liq}}^{\mathrm{EFG}} \simeq[(2-\pi / 2) P+(2 \sqrt{3}-\pi) H] R^{2}
$$

Far away from regime FG $(P \gg H \gg R)$, one has:

$$
\Omega_{\mathrm{liq}}^{\mathrm{E}} \simeq(2-\pi / 2) P R^{2}
$$

The above distinction between the pancake regime and the floor tile regime, based solely on the overall shape of the bubbles (rounded edges versus sharp edges), is sufficient to provide the results of Eqs. (3) and (7) concerning the liquid volume per bubble in the various regimes (see Table III).

But for other purposes, in particular concerning dilatancy [4], one must refine the geometrical description of the bubbles in such a GG foam in the pancake regime. That is the scope of the remaining of the present Section.

\section{B. Pancake regime with elliptical cross-section}

Only numerical tools such as Surface Evolver [8] can provide an exact description of the bubbles when a more thorough geometrical description than the above approximation is needed.
In the present paragraph devoted to the pancake regime, we consider an intermediate approximation based on an elliptical description of the pseudo Plateau border cross-section and derive various geometrical properties. A similar derivation (with a simpler geometrical description) was carried out recently [9] in the context of coarsening experiments.

The shape of an inter-bubble film is a surface whose total curvature is related to the pressure difference between both bubbles:

$$
\frac{1}{\rho_{1}}+\frac{1}{\rho_{2}}=\frac{p_{B}-p_{A}}{2 \gamma}
$$

where $\rho_{1}$ and $\rho_{2}$ are the principal radii of curvature, $p_{A}$ and $p_{B}$ are the gas pressure in bubbles $\mathrm{A}$ and $\mathrm{B}$, and where the surface tension of the film is assumed to be equal to twice that of a single interface, $\gamma$. In the present work, for simplicity we assume that all bubbles have equal pressures, so that the films are planar: $\rho_{1}=\rho_{2}=\infty$.

Since the films are assumed planar, the pseudo Plateau borders are circular in cross-section (radius $R_{\mathrm{ps}}$, see Fig. 1 bottom right) and the total curvature of the gasliquid interface is therefore $1 / R_{\mathrm{ps}}$.

In the Plateau border region, the meniscus is roughly toroidal in shape: the Plateau border is axisymmetric (radius $R$ ), and the interface is curved more strongly in the vertical direction (with a radius of curvature close to $H / 2)$. Let $r(z)$ be the equation for its surface, in cylindrical coordinates as measured from its axis of symmetry. The constant total curvature condition reads:

$$
\frac{-r^{\prime \prime}}{\left(1+r^{\prime 2}\right)^{3 / 2}}+\frac{1}{r\left(1+r^{\prime 2}\right)^{1 / 2}}=\text { const }=\frac{1}{R_{\mathrm{ps}}}
$$

where $r^{\prime}=\mathrm{d} r / \mathrm{d} z$ and $r^{\prime \prime}=\mathrm{d}^{2} r / \mathrm{d} z^{2}$. The first term is the curvature within a meridian plane, while the second term is the curvature within a plane perpendicular to the meridian curve. The value of the constant is chosen so as to match the total curvature found for the same gasliquid interface in the pseudo Plateau borders, namely $1 / R_{\mathrm{ps}}$.

Of course, Eq. (10) for the surface shape can be solved numerically. Here, we shall simply take the limit $R \gg H$ (which corresponds to asymptotic regimes $\mathrm{AB}$ and $\mathrm{CD}$ ), where bubbles are not pressed very strongly against one another. This has two consequences: (i) the interbubble films are very small in height $\left(H-2 R_{\mathrm{ps}} \ll H\right)$ and (ii) the shape of the interface in the Plateau border can be approximated as elliptical in cross-section.

With this elliptical approximation (see Fig. 1 top right), the various geometrical parameters can be obtained very easily. At mid-height, the azimuthal radius of curvature is simply the Plateau border radius $R$. Since the total curvature is known to be $1 / R_{\mathrm{ps}}$, the radius of curvature $r_{1}$ in the meridian plane at mid-height can be obtained through:

$$
\frac{1}{r_{1}}+\frac{1}{R}=\frac{1}{R_{\mathrm{ps}}}
$$


Since the length of the greater semi-axis of the ellipse is simply $H / 2$, one has:

$$
(H / 2)^{3}=r_{1}^{2} R_{\mathrm{ps}}
$$

With these two equations and keeping in mind that $R \gg$ $H$, one can then deduce the various lengths that appear on Fig. 1 (top right), including the smaller semi-axis $D_{R}$ given by $D_{R}^{3}=R_{\mathrm{ps}}^{2} r_{1}$ :

$$
\begin{aligned}
r_{1} & \simeq \frac{H}{2}\left[1+\frac{1}{6} \frac{H}{R}\right] \\
R_{\mathrm{ps}}^{\mathrm{ABCD}} & \simeq \frac{H}{2}\left[1-\frac{1}{3} \frac{H}{R}\right] \\
D_{R} & \simeq \frac{H}{2}\left[1-\frac{1}{6} \frac{H}{R}\right]
\end{aligned}
$$

Note that the current description in terms of Plateau borders with an elliptical cross-section and pseudo Plateau borders with an circular cross-section is not entirely consistent: the crossover between both regions implies that the interface adopt an intermediate shape near the cuspy corners of the Plateau border. In the present limit $R \gg H$, this discrepancy can be safely neglected. Similarly, the elliptical approximation for the solution of Eq. (10) is sufficient. Nevertheless, all subsequent results will not be expressed beyond the first order in $H / R$.

The height $\varepsilon$ of the interbubble film is given by:

$$
\varepsilon=H-2 R_{\mathrm{ps}} \simeq \frac{1}{3} \frac{H^{2}}{R}
$$

Note that with a stronger geometrical assumption (circular rather than elliptical cross-section for the torus in the Plateau border), Marchalot et al. [9] obtain almost the same result. Only the numerical prefactor differs $(1 / 2$ in Ref. [9] instead of $1 / 3$ in Eq. (16) above).

\section{Liquid volume per pancake}

Let us now refine Eq. (2) with the elliptical approximation presented above.

The first term, $(2-\pi / 2) P R_{\mathrm{ps}}^{2}$, corresponds to the pseudo Plateau borders (light grey regions of Fig. 1 1 when seen from above) and already assumes that their crosssection is circular. Hence, this part of the equation remains correct in the refined description, provided the value of $R_{\mathrm{ps}}$ is taken from Eq. (14) and provided we subtract the part of the perimeter that corresponds to the Plateau borders:

$$
\frac{4-\pi}{8} H^{2}(P-2 \pi R)\left[1-\frac{2 H}{3 R}\right]
$$

where $2 \pi R$ is the sum of the six Plateau border perimeters of a typical bubble (i.e., the total length of the separation between light grey and black regions in Fig. 11).

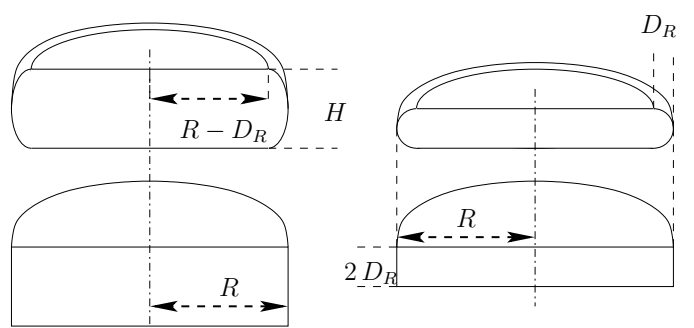

FIG. 3: Contribution to the liquid in a Plateau border. Within a Plateau border, the gas-liquid interface has roughly the shape of the outer surface of an elliptical torus (top left). Hence, part of the liquid in the Plateau borders of a bubble consists in the region between such an elliptical torus and the circular wall of a vertical cylinder (bottom left) with the same outer radius $R$. To calculate the volume of this contribution more easily, we reduce the height of both the torus (top right) and the cylinder (bottom right) by such a factor that the torus has a circular cross-section.

The second term, $(2 \sqrt{3}-\pi) R^{2} H$, still correctly represents the part of the Plateau borders that spans the entire gap $H$ between both plates (black regions on Fig. 11). We must add, however, the volume of liquid located above and below the elliptical region of the interface (which corresponds to the curved part of the grey ribbons that surround the black Plateau border core regions). If we put together all these Plateau border edges for one single bubble, we obtain a fully axisymmetric region of thickness $H$ that is described on Fig. 3 (left). Its volume can be deduced from that of a rounded flat cylinder with thickness $2 D_{R}$ and with a toroidal edge (see Fig. 3 right). The volume of such a rounded flat cylinder (top right) can be expressed as:

$$
2 D_{R} \pi\left(R-D_{R}\right)^{2}+2 \pi\left(R-D_{R}\right) \frac{\pi D_{R}^{2}}{2}+\frac{4 \pi D_{R}^{3}}{3}
$$

where the first term is the volume of a flat cylinder with radius $R-D_{R}$ and height $2 D_{R}$, the second term is that of a semi-cylinder whose radius is $D_{R}$ and whose length is the perimeter of the first flat cylinder. Finally, the third term is the correction that accounts for the curvature of the semi-cylinder, and it is equal to the volume of a sphere of radius $D_{R}$. Subtracting these terms from the volume $2 D_{R} \pi R^{2}$ of the outer flat cylinder (see Fig. 3 bottom right) and multiplying this result by a factor $H /\left(2 D_{R}\right)$, we obtain the desired liquid contribution:

$$
\frac{H}{2 D_{R}}\left[\pi(4-\pi) D_{R}^{2} R-\frac{\pi(10-3 \pi)}{3} D_{R}^{3}\right]
$$

Combining this with Eq. (17) and with the second term of Eq. (2), we finally obtain:

$$
\begin{array}{r}
\Omega_{\mathrm{liq}} \simeq \frac{4-\pi}{8} H^{2} P+(2 \sqrt{3}-\pi) R^{2} H-\frac{4-\pi}{12} \frac{H^{3} P}{R} \\
+\frac{\pi}{12} H^{3}+\frac{\pi(10-3 \pi)}{72} \frac{H^{4}}{R}
\end{array}
$$


The relative magnitude of the first three terms changes between regimes $\mathrm{AB}$ and $\mathrm{CD}$ (see Fig. 2), while the fourth and fifth terms are always negligible in both regimes:

$$
\begin{aligned}
& \text { regime } \mathrm{AB}: \quad T_{2} \gg T_{1} \gg T_{3} \gg T_{4} \gg T_{5} \\
& \text { regime } \mathrm{CD}: T_{1} \gg\left(T_{2} \text { and } T_{3}\right) \gg T_{4} \gg T_{5}
\end{aligned}
$$

Because the fourth and fifth terms are always negligible, we shall retain only the first three terms in Eq. (20):

$$
\Omega_{\mathrm{liq}}^{\mathrm{ABCD}} \simeq \frac{4-\pi}{8} H^{2} P\left[1-\frac{2 H}{3 R}\right]+(2 \sqrt{3}-\pi) R^{2} H
$$

which refines the result of Eq. (3). In other words, we use the elliptical approximation to obtain the value $R_{\mathrm{ps}}$ of the pseudo Plateau border radius, see Eq. (14). But we then calculate the volume as if the shape of the interface in the Plateau borders were identical to that in the pseudo Plateau borders, i.e., with circular (radius $R_{\mathrm{ps}}$ ) rather than elliptical sections (see Fig. 1 bottom right). This is legitimate because in the limit of small liquid fractions $(\phi \ll 1)$, the total length of the Plateau borders $(2 \pi R)$ is much smaller than that of the pseuso Plateau borders $(P)$.

\section{Liquid volume fraction}

From Eqs. (17) and (22), we derive the liquid volume fraction $\phi=\Omega_{\text {liq }} /\left(\mathcal{A}_{\text {tot }} H\right)$ in the foam, both in the pancake regime and in the floor tile regime:

$$
\begin{aligned}
\phi^{\mathrm{ABCD}} \simeq & \frac{4-\pi}{8} \frac{H P}{\mathcal{A}_{\mathrm{tot}}}\left[1-\frac{2 H}{3 R}\right] \\
& +(2 \sqrt{3}-\pi) \frac{R^{2}}{\mathcal{A}_{\mathrm{tot}}} \\
\phi^{\mathrm{EFG}} \simeq & \frac{4-\pi}{2} \frac{R^{2} P}{\mathcal{A}_{\mathrm{tot}} H}+(2 \sqrt{3}-\pi) \frac{R^{2}}{\mathcal{A}_{\mathrm{tot}}}
\end{aligned}
$$

The corresponding asymptotic values of $\phi$ in all subregimes are indicated in Table [1]. It is useful to express the liquid fraction in terms of the volume $\Omega$ of the bubble itself. Using $\phi /(1-\phi)=\mathcal{A}_{\text {tot }} H \phi / \Omega$ and the total volume of the bubble and liquid $\mathcal{A}_{\text {tot }} H=\Omega+\Omega_{\text {liq }}$, the above equations become:

$$
\begin{aligned}
\frac{\phi^{\mathrm{ABCD}}}{1-\phi^{\mathrm{ABCD}}} \simeq & \frac{4-\pi}{8} \frac{H^{2} P}{\Omega}\left[1-\frac{2 H}{3 R}\right] \\
& +(2 \sqrt{3}-\pi) \frac{R^{2} H}{\Omega} \\
\frac{\phi^{\mathrm{EFG}}}{1-\phi^{\mathrm{EFG}}} \simeq & \frac{4-\pi}{2} \frac{R^{2} P}{\Omega}+(2 \sqrt{3}-\pi) \frac{R^{2} H}{\Omega}
\end{aligned}
$$

\section{E. Specific surface area}

With the same approximation as above, let us calculate the total surface area of a bubble. As it is directly related to the foam energy, it is useful when deriving the elastic modulus of the foam [5].

The contribution from the top and the bottom of a bubble to the in-plane component is the white region of the top view in Fig. 11:

$$
2\left\{\mathcal{A}_{\mathrm{tot}}-\left[2 \sqrt{3} R^{2}-\pi\left(R-D_{R}\right)^{2}\right]-(P-2 \pi R) R_{\mathrm{ps}}\right\}
$$

In this expression, the term $-2 \sqrt{3} R^{2}$ corresponds to one third of the dashed-line triangle with edge length $2 R$ in Fig. 1 and similarly for the other Plateau borders of the bubble. The third term, $\pi\left(R-D_{R}\right)^{2}$, corresponds to the dark grey sector in the triangle. The next term, $-(P-$ $2 \pi R) R_{\mathrm{ps}}$, corresponds to the wall surface area that does not touch the bubble along the pseudo Plateau borders (light grey ribbon).

Making the approximation $D_{R} \simeq R_{\mathrm{ps}}$ in the Plateau border region as mentioned at the end of Paragraph $\mathbb{I I G}$ and using $R_{\mathrm{ps}} \ll P$, this becomes:

$$
2 \mathcal{A}_{\mathrm{tot}}-(4 \sqrt{3}-2 \pi) R^{2}-2 P R_{\mathrm{ps}}
$$

The vertical films contribution is:

$$
P\left(H-2 R_{\mathrm{ps}}\right)
$$

The contribution from the menisci, considered as circular quarter cylinders with radius $R_{\mathrm{ps}}$, can be written as:

$$
2 P \int_{0}^{\frac{\pi}{2}} R_{\mathrm{ps}} \mathrm{d} \theta=\pi P R_{\mathrm{ps}}
$$

Hence, the specific surface area $\Sigma$ of the foam, equal to the bubble total surface area divided by the total volume $\mathcal{A}_{\text {tot }} H$, includes all three contributions above:

$$
\begin{aligned}
\mathcal{A}_{\mathrm{tot}} H \Sigma \simeq & 2 \mathcal{A}_{\mathrm{tot}}-(4 \sqrt{3}-2 \pi) R^{2}-2 P R_{\mathrm{ps}} \\
& +P\left(H-2 R_{\mathrm{ps}}\right)+\pi P R_{\mathrm{ps}} \\
\Sigma \simeq & \frac{2}{H}-(4 \sqrt{3}-2 \pi) \frac{R^{2}}{\mathcal{A}_{\mathrm{tot}} H} \\
& +\frac{P}{\mathcal{A}_{\mathrm{tot}}}-(4-\pi) \frac{P R_{\mathrm{ps}}}{\mathcal{A}_{\mathrm{tot}} H}
\end{aligned}
$$

The final result is then derived both in the pancake regime where $R_{\mathrm{ps}}$ is given by Eq. (14) and where $R_{\mathrm{ps}} \ll R$ (regimes AB and CD of Fig. 2) and in the floor tile regime where $R_{\mathrm{ps}}=R$ (regimes $\mathrm{E}$ and $\mathrm{FG}$ ):

$$
\begin{aligned}
\Sigma_{\mathrm{ABCD}} \simeq & \frac{2}{H}-(4 \sqrt{3}-2 \pi) \frac{R^{2}}{\mathcal{A}_{\mathrm{tot}} H} \\
& +\frac{\pi-2}{2} \frac{P}{\mathcal{A}_{\mathrm{tot}}}+\frac{4-\pi}{6} \frac{P H}{\mathcal{A}_{\mathrm{tot}} R} \\
\Sigma_{\mathrm{EFG}} \simeq & \frac{2}{H}+\frac{P}{\mathcal{A}_{\mathrm{tot}}}-(4-\pi) \frac{P R}{\mathcal{A}_{\mathrm{tot}} H} \\
& -(4 \sqrt{3}-2 \pi) \frac{R^{2}}{\mathcal{A}_{\mathrm{tot}} H}
\end{aligned}
$$

These results are reproduced in Table II. 


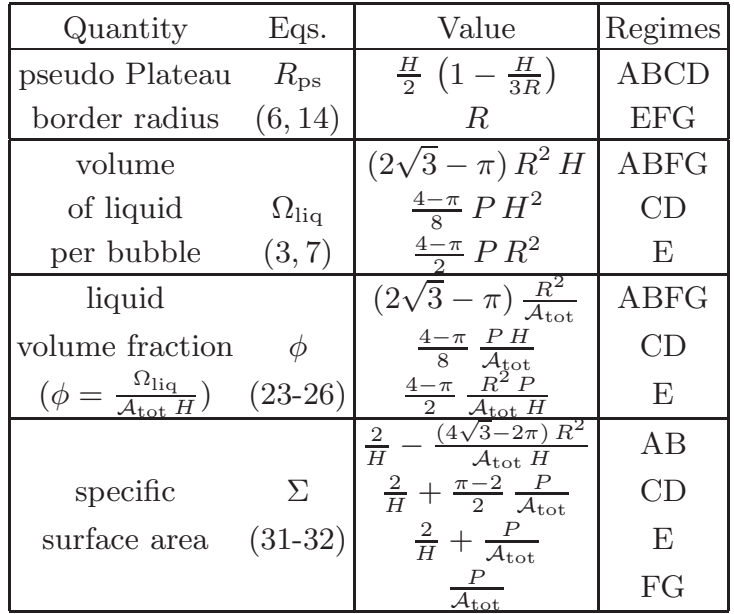

TABLE II: Geometrical properties of a two-dimensional glassglass foam. The numbers refer to the relevant series of equations and the letters to the regimes of Fig. 2 pancake regime $(\mathrm{AB}$ and $\mathrm{CD})$ and floor tile regime (E and $\mathrm{FG}$ ). In each expression, the terms are ordered by decreasing magnitudes.

\section{PREDICTED NON-TRIVIAL DISCONTINUITIES IN THREE EXPERIMENTAL CONDITIONS: THE BULIMIC PLATEAU BORDERS}

We shall now analyse the geometrical quantities derived in the previous section and show that they predict non-trivial discontinuities.

When preparing an undeformed 2D GG foam, the experimental conditions determine the value of three independent variables, for instance the liquid fraction $\phi$, the bubble volume $\Omega$ and the cell thickness $H$.

Once these quantities are fixed, the bubble perimeter $P$ depends principally on $\Omega$ and $H$, with the scaling

$$
\Omega \simeq P^{2} H,
$$

since $P^{2}$ scales like the bubble surface area $\mathcal{A}_{\text {tot }}$. It also depends more weakly on the degree of disorder of the foam and on the bubble size distribution. Finally, it is also sensitive to the foam deformation, and this may be at the origin of static dilatancy, as we show elsewhere $\left[\begin{array}{l}\text {. } \\ \text {. }\end{array}\right.$

The Plateau border radius $R$ also results from $\phi, \Omega$ and $H$ and from the foam equilibrium.

Then other quantities, such as those derived in Section III above, are also determined.

\section{A. Description and origin of the transition}

The above calculations have a striking consequence: an intrinsic instability of regime CD leads to discontinuities in the dimension of the Plateau borders when varying continuously $\phi, H$ or $P$.

In Section III, for simplicity, the liquid fraction $\phi$ was expressed in terms of $P, H$ and $\Omega$ or $\mathcal{A}_{\text {tot }}$. It appears that in regime $\mathrm{CD}, \phi$ does not depend on $R$ (see Table III). A more refined analysis, based on Eq. (25), reveals that there is a slight dependence of $\phi$ on $R$ :

$$
\frac{8}{4-\pi} \frac{\Omega \phi}{H^{2} P}-1 \simeq \frac{8(2 \sqrt{3}-\pi)}{4-\pi} \frac{R^{2}}{H P}-\frac{2}{3} \frac{H}{R}
$$

which shows that $\phi \simeq \frac{4-\pi}{8} \frac{H^{2} P}{\Omega}$ changes only by a factor close to unity when $R$ changes from small values comparable to $H$ to much larger values comparable to $\sqrt{H P}$. In other words, the Plateau border radius $R$ depends very strongly on the other parameters in this region. When $H^{4 / 3} P^{2 / 3} \ll R^{2} \ll H P$, Eq. (25) yields:

$$
R \simeq \sqrt{\frac{\Omega}{(2 \sqrt{3}-\pi) H}\left(\phi-\frac{4-\pi}{8} \frac{H^{2} P}{\Omega}\right)}
$$

Similarly, when $H \ll P \ll H^{2 / 3} P^{1 / 3}$ :

$$
R \simeq \frac{\frac{2}{3} H}{1-\frac{8}{4-\pi} \frac{\phi \Omega}{H^{2} P}}
$$

Thus, abrupt transitions can thus occur for the Plateau border radius $R$ without any significant changes in other characteristic foam parameters.

This effect can be described in physical terms as follows. When the foam goes across regime $\mathrm{CD}$, the fact that the Plateau border radius $R$ is the only variable that varies significantly, indicates that some liquid is exchanged between the Plateau borders and the pseudo Plateau borders. But as can be seen from Table If, in regime $\mathrm{CD}$ the volume of the Plateau borders is small compared to that of the pseudo Plateau borders. Hence, this exchange of liquid is of limited relative importance for the foam, which explains why other variables are affected only marginally.

\section{B. Three experimental situations}

Let us now imagine three experimental situations where the present "bulimia" effect should be apparent in a GG-foam. The foam should be subjected to: (1) progressive drying, (2) coarsening (through film rupture or Oswald ripening), (3) progressive squeezing. Again, let us insist on the fact that the present predictions rely on the assumption that the foam is almost at equilibrium and that there is no spatial inhomogeneity in the foam.

\section{Drying foam}

Let us consider a GG-foam being progressively dried (decreasing $\phi$ ), with constant bubble thickness $H$ and volume $\Omega$, hence almost constant perimeter $P$.

Starting near the liquid-solid transition, with liquid fraction $\phi$ comparable to unity, the foam evolves vertically upwards in the diagram of Fig. Ha, at first in regime 


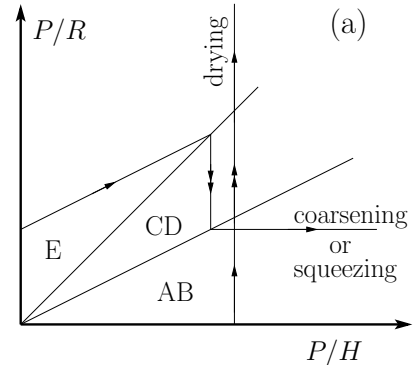

(c) coarsening

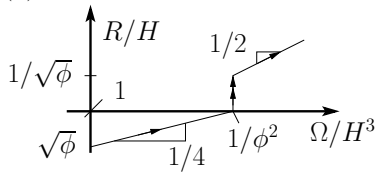

d) squeezing

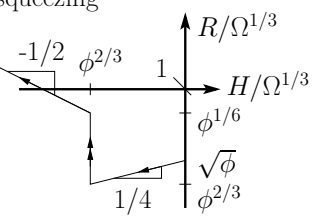

FIG. 4: Structural evolution of a 2D GG foam undergoing three different experiments. The rapid evolution through regime $\mathrm{CD}$ is symbolized by double arrows. (a) Upon drying, the foam goes from regime $\mathrm{AB}$ to regime $\mathrm{E}$ (in the notations of Fig. 2). By contrast, when it is squeezed or when it coarsens spontaneously, it evolves from regime $\mathrm{E}$ to regime $\mathrm{AB} .(b-d)$ Schematic Plateau border radius evolution during three types of experiments. (b) Drying: constant $P$ and $H$, decreasing $\phi$. (c) Coarsening: constant $\phi$ and $H$, increasing $\Omega$ and $P$. (d) Squeezing: constant $\phi$ and $\Omega$, decreasing $H$ and increasing $P$.

$\mathrm{AB}$, then in regime $\mathrm{CD}$ (very rapidly as a result of the strong variation of $R$ mentioned in Section [IIA), and finally in regime $\mathrm{E}$.

The variations of the Plateau border radius $R$ are plotted on Fig. Ab as a function of the liquid fraction $\phi$. Initially, it is comparable to the bubble perimeter, it decreases as $\sqrt{\phi}$ in regime $\mathrm{AB}$, it then jumps down through regime $\mathrm{CD}$ to reach roughly $H / 2$, then it again decreases as $\sqrt{\phi}$ in regime $\mathrm{E}$ :

$$
\begin{aligned}
R_{\phi \simeq 1} & \simeq P \\
R_{\mathrm{AB}} & \simeq P \sqrt{\phi} \\
H & \lesssim R_{\mathrm{CD}} \lesssim \sqrt{P H} \\
R_{\mathrm{E}} & \simeq \sqrt{P H} \sqrt{\phi}
\end{aligned}
$$

Of course, Eq. (33) must be used if the thickness $H$ and the bubble volume $\Omega$ are prefered to the perimeter $P$ as constant parameters.

\section{Coarsening foam}

Let us now consider a GG-foam that coarsens progressively (increasing typical bubble volume $\Omega$ and perimeter $P$ ), with constant thickness $H$ and liquid fraction $\phi$. This coarsening can result from various phenomena [1. When inter-bubble film are not very stable, neighbouring bubbles may coalesce and the average bubble size increases. Alternatively, coarsening can result from continuous Oswald ripening: gas diffusion between neighbouring bubbles leads to a net flux from small bubbles to large bubbles as a result of the larger pressure in the smaller bubbles; large bubbles grow even larger, small bubbles vanish and the size distribution evolves to larger length scales.

Starting near the 2D stability transition, with a horizontal size (or perimeter $P$ ) comparable to the thickness $H$, the foam evolves as shown on the diagram of Fig. A 4 a, at first in regime $\mathrm{E}$, then in regime $\mathrm{CD}$ (very rapidly as a result of the strong variation of $R$ mentioned in Section III A), and finally horizontally in regime AB.

The variations of the Plateau border radius $R$ are plotted on Fig. Ac as a function of the bubble volume $\Omega$. It increases like $\Omega^{1 / 4}$ in regime $E$, it then jumps up through regime $\mathrm{CD}$ from $H / 2$ to roughly $H / \sqrt{\phi}$, then it again increases like $\sqrt{\Omega}$ in regime $\mathrm{AB}$ :

$$
\begin{aligned}
R_{\mathrm{E}} & \simeq(\Omega H)^{1 / 4} \sqrt{\phi} \\
H & \lesssim R_{\mathrm{CD}} \lesssim H / \sqrt{\phi} \\
R_{\mathrm{AB}} & \simeq \sqrt{\Omega \phi / H}
\end{aligned}
$$

Of course, Eq. (33) must be used if the perimeter $P$ is prefered to the bubble volume $\Omega$ as the main variable.

In coarsening experiments such as those conducted by Marchalot et al. [9], a (delicate) measurement of the Plateau border radius $R$ will be necessary to observe the phenomenon described above clearly.

\section{Squeezing a foam}

Let us now consider a GG-foam that is being squeezed progressively (decreasing thickness $H$ ) at constant bubble volume $\Omega$ and liquid fraction $\phi$.

Starting near the 2D stability transition, with a horizontal size (or perimeter $P$ ) comparable to the thickness $H$, the foam evolves as shown on the diagram of Fig. 4a, at first in regime $\mathrm{E}$, then in regime $\mathrm{CD}$ (very rapidly as a result of the strong variation of $R$ mentioned in Section III A), and finally horizontally in regime AB.

The variations of the Plateau border radius $R$ are plotted on Fig. 4 $\mathrm{d}$ as a function of the sample thickness $H$. It is non-monotonic: it decreases like $H^{1 / 4}$ in regime $\mathrm{E}$, it then jumps up through regime $\mathrm{CD}$, then it increases like $1 / \sqrt{H}$ in regime $\mathrm{AB}$ :

$$
\begin{aligned}
R_{\mathrm{E}} & \simeq(\Omega H)^{1 / 4} \sqrt{\phi} \\
\Omega^{1 / 3} \phi^{2 / 3} & \lesssim R_{\mathrm{CD}} \lesssim \Omega^{1 / 3} \phi^{1 / 6} \\
R_{\mathrm{AB}} & \simeq \sqrt{\Omega \phi / H}
\end{aligned}
$$

Of course, Eq. (33) must be used if the perimeter $P$ is prefered to the thickness $H$ as the main variable. 


\section{CONCLUSION}

By examining the geometry of 2D "GG"-foams (located between two parallel solid plates), we found that these foams should display a rather strong "bulimia" transition where Plateau borders swallow (going from retime $\mathrm{E}$ to regime $\mathrm{AB}$ ) a large amount of liquid when the foam parameters are varied in a narrow region. This effect, which is specific to $2 \mathrm{D}$ "GG"-foams, should be observable in at least three types of experiments, for which we provided first-order predictions. Of course, there may be other experimental situations. Let us insist again on the fact that the present predictions are restricted to foams almost at equilibrium and with no spatial inhomogeneity. This "bulimia" effect should affect other phenomena, such as dilatancy; this is discussed elsewhere [4, 5].

\section{Acknowledgements}

We gratefully acknowledge fruitful discussions with participants of the GDR 2983 Mousses (CNRS), in particular with Julien Marchalot, Marie-Caroline Jullien and Isabelle Cantat about the Plateau border geometry. This work was supported by the Agence Nationale de la Recherche (ANR05).
[1] D. Weaire and S. Hutzler, The Physics of Foams (Oxford University Press, 1999).

[2] D. Weaire, Curr. Opin. Coll. Interf. Sci. 13, 171 (2008), ISSN 1359-0294, URL http://www.sciencedirect. om/science/article/B6VRY-4R40SMC-3/2/ 51424d6812da808741b7ada7043d2123.

[3] R. Hohler and S. Cohen-Addad, J. Phys.: Condens. Matter 17, 1041 (2005).

[4] P. Rognon, F. Molino, and C. Gay, in preparation (2008).

[5] P. Rognon, F. Molino, and C. Gay, in preparation (2008).
[6] D. Weaire and S. Hutzler, Phil. Mag. 83, 2747 (2003).

[7] F. Rioual, S. Hutzler, and D. Weaire, Coll. Surf. A 263, 117 (2005).

[8] K. Brakke, Expt. Math. 1, 141 (1992).

[9] J. Marchalot, J. Lambert, I. Cantat, P. Tabeling, and M.-C. Jullien, EPL (Europhysics Letters) 83, 64006 (6pp) (2008), URL http://stacks .iop.org/0295-5075/ $83 / 64006$ 